\title{
Frontières
}

\section{Le programme Intervention-suicide en milieu scolaire primaire}

\author{
Évaluation de l'implantation en Gaspésie et aux \\ Îles-de-la-Madeleine
}

\section{Marc Daigle et Rodrigue Gallagher}

Volume 21, numéro 1, automne 2008

Prévenir le suicide

URI : https://id.erudit.org/iderudit/037880ar

DOI : https://doi.org/10.7202/037880ar

Aller au sommaire du numéro

Éditeur(s)

Université du Québec à Montréal

ISSN

1180-3479 (imprimé)

1916-0976 (numérique)

Découvrir la revue

Citer cet article

Daigle, M. \& Gallagher, R. (2008). Le programme Intervention-suicide en milieu scolaire primaire : évaluation de l'implantation en Gaspésie et aux

Îles-de-la-Madeleine. Frontières, 21(1), 107-112.

https://doi.org/10.7202/037880ar
Résumé de l'article

Cet article présente les résultats de l'évaluation de l'implantation du programme Intervention-suicide en milieu scolaire primaire. Les principales forces de ce programme résident dans sa spécificité, l'articulation du travail du personnel scolaire et des intervenants professionnels, la motivation, l'engagement et la diligence de ses partenaires, le professionnalisme des intervenants, l'expertise et la motivation particulière du responsable du programme, la qualité de la documentation disponible, la qualité des formations et sensibilisations dispensées, l'utilisation des forces d'intervention de la santé et de l'éducation, la diversité des expertises et expériences chez les intervenants, l'ouverture à la critique et la modération dans l'interprétation du phénomène suicidaire dans les actions à prendre. Des faiblesses relatives sont cependant notées au regard des aspects suivants : le partage des

responsabilités, le manque d'information explicite dans les guides sur les objectifs, la structure et les fondements théoriques du programme, les formations trop courtes offertes aux intervenants, la dilution de l'expertise des intervenants, l'isolement de certains de ceux-ci, l'absence fréquente de leadership dans les équipes de secteur, et le décalage dans la durée de la formation clinique des intervenants.
Ce document est protégé par la loi sur le droit d'auteur. L'utilisation des services d'Érudit (y compris la reproduction) est assujettie à sa politique d'utilisation que vous pouvez consulter en ligne.

https://apropos.erudit.org/fr/usagers/politique-dutilisation/ 
Résumé

Cet article présente les résultats de l'évaluation de l'implantation du programme Intervention-suicide en milieu scolaire primaire. Les principales forces de ce programme résident dans sa spécificité, l'articulation du travail du personnel scolaire et des intervenants professionnels, la motivation, l'engagement et la diligence de ses partenaires, le professionnalisme des intervenants, l'expertise et la motivation particulière du responsable du programme, la qualité de la documentation disponible, la qualité des formations et sensibilisations dispensées, l'utilisation des forces d'intervention de la santé et de l'éducation, la diversité des expertises et expériences chez les intervenants, l'ouverture à la critique et la modération dans I'interprétation du phénomène suicidaire dans les actions à prendre. Des faiblesses relatives sont cependant notées au regard des aspects suivants: le partage des responsabilités, le manque d'information explicite dans les guides sur les objectifs, la structure et les fondements théoriques du programme, les formations trop courtes offertes aux intervenants, la dilution de l'expertise des intervenants, l'isolement de certains de ceux-ci, l'absence fréquente de leadership dans les équipes de secteur, et le décalage dans la durée de la formation clinique des intervenants.

Mots clés: suicide - prévention scolaire - jeune - évaluation.

\section{Abstract}

The article documents the results of the evaluation of the implementation of a suicide intervention program in primary schools in a remote region of Quebec. The main strengths of the program include: its specificity; task coordination between school staff and professional practitioners; highly motivated, engaged and diligent partners; professionalism of practitioners; expertise and personal motivation of program head; quality of documentation available; quality of training and awareness-raising activities provided; use of power of intervention of healthcare and education systems; diversity of practitioner expertise and experience; openness to criticism and moderate interpretation of suicide phenomenon in actions to take. Certain relative weaknesses are observed in terms of sharing of responsibilities, lack of explicit information on objectives in program guides, program's structure and theoretical foundation, overly short duration of training offered to practitioners, dilution of practitioner expertise, isolation of some practitioners, frequent absence of leadership in sector teams, and differences in duration of clinical training of practitioners.

Keywords: suicide - prevention school - child - evaluation.

\section{Le programme Intervention- suicide en milieu scolaire primaire Évaluation de I'implantation en Gaspésie et aux Îles-de-la-Madeleine}

\author{
Marc Daigle, Ph. D. \\ professeur, Département de psychologie, \\ Université du Québec à Trois-Rivières, \\ membre chercheur, CRISE, chercheur titulaire, \\ Institut Philippe-Pinel de Montréal.
}

Rodrigue Gallagher, M.A., agent de planification, programmation et recherche, Direction de santé publique et de l'évaluation, ASSS de Chaudière-Appalaches.

Comment est-il possible de comprendre ce qu'est un comportement suicidaire, surtout chez un enfant? L'expérience clinique et la recherche scientifique peuvent répondre en partie à cette question. Cependant, un Gaspésien de 10 ans qui en est rescapé nous explique lui-même, du haut de la grande sagesse et naïveté propres à son âge, ce qu'il en est: "Je voulais arrêter d'avoir mal en dedans.»Or, le programme Intervention-suicide en milieu scolaire primaire, tel qu'il a été présenté dans un autre article de ce numéro de Frontières, semble innover dans cette tâche de diminuer, autrement que par le passage à l'acte auto-agressif, la souffrance de certains enfants. Ce deuxième article résume l'évaluation qui a été faite de ce programme (voir Daigle, 2007).
Le programme évalué ici est donc né de l'effort d'une communauté pour prendre en charge la souffrance de certains enfants, laquelle peut s'accompagner d'idéations et même de comportements suicidaires. En parallèle, le programme veut aussi prendre en charge la souffrance des enfants dont des proches se sont suicidés. Notons que les écoles primaires sont un lieu fort propice à l'intervention, surtout dans le cas qui nous intéresse ici, soit les jeunes enfants. Néanmoins, soulignons au passage que, encore une fois, c'est sur l'école que nous nous reposons alors que toute la société se devrait d'être mobilisée, comme le proclame le proverbe africain inscrit en grandes lettres sur la façade d'une école gaspésienne: "Il faut tout un village pour éduquer un enfant ${ }^{1}$.»Comme le souligne D'Amours (2001, p. 27), «les attentes sociales relatives à l'école sont extrêmement grandes. On veut que les jeunes sachent bien écrire, sachent bien compter, mais les aspects de la prévention et les attentes sur le plan scolaire ne sont pas toujours faciles à concilier». Or, selon ses promoteurs, le programme qui est évalué ici se veut justement un bon exemple de conciliation et d'intégration en milieu scolaire. 


\section{RECENSION DES ÉCRITS DANS LE DOMAINE}

Pour évaluer ce programme qui se veut novateur, l'évaluateur a dû développer des approches originales qui tiennent compte justement de ses particularités. Les recherches et évaluations déjà connues dans la littérature scientifique ne répondaient pas à l'ensemble des besoins d'évaluation. Toutefois, l'évaluateur s'est inspiré de ce qui avait déjà été fait dans trois domaines parallèles: 1) la prévention du suicide chez les jeunes, 2) la prévention du suicide en milieu scolaire et 3 ) l'évaluation des programmes de formation ou de sensibilisation, en général.

\section{PRÉVENTION DU SUICIDE \\ CHEZ LES JEUNES}

Un colloque tenu en 2000 à l'Université du Québec à Montréal (ministère de la Santé et des Services sociaux, 2001), sous l'égide du Centre de recherche et d'intervention sur le suicide et l'euthanasie (CRISE), voulait couvrir l'ensemble de la question de la prévention du suicide chez les jeunes. De façon incontournable, ce colloque s'intéressait cependant plus particulièrement aux quelques endroits où des jeunes à risque suicidaire peuvent le plus facilement être rejoints: les centres jeunesse, les cliniques et, tout naturellement, les écoles. En effet, comme il a été souligné plus haut, l'école est le milieu de vie où les jeunes peuvent le plus facilement être rejoints. L'Avis scientifique sur la prévention du suicide chez les jeunes de l'Institut national de santé publique du Québec (Julien et al., 2004) a aussi couvert l'ensemble de la question avec rigueur, en insistant plus cette fois sur l'aspect évaluatif (Julien, 2003). Ces quelques documents placent déjà le cadre général où s'est située l'évaluation rapportée ici, notamment au niveau des lieux possibles de la prévention et de la nécessité d'en évaluer les divers aspects.

\section{PRÉVENTION DU SUICIDE}

EN MILIEU SCOLAIRE

D'Amours (2001, p. 25-26) situe les contributions possibles de l'école québécoise à la prévention du suicide à l'intérieur de cinq champs d'action plus large: 1 ) développement des compétences sociales, 2) offre de services d'aide et de soutien aux élèves en difficulté, 3) sensibilisation et formation pour le personnel scolaire, 4) formation des jeunes à l'entraide et 5) transmission d'information aux élèves et à leurs parents sur la maladie mentale, les signes précurseurs des conduites suicidaires et l'homosexualité. On peut penser que c'est pour toutes ces raisons que les Commissions scolaires (CS) de la
Gaspésie-Îles-de-la-Madeleine avaient déjà un programme pour le milieu secondaire avant l'implantation du programme qui est maintenant spécifique au niveau primaire.

L'école est effectivement un milieu de vie où les jeunes passent une grande partie de leur temps quotidien et où leur développement se poursuit dans un certain ordre des choses, habituellement loin des drames. De plus, il s'agit là d'un milieu hautement structuré où les intervenants de divers ordres ont la possibilité d'exercer une certaine influence bénéfique, notamment pour ce qui est de la prévention du suicide. Inversement, l'école peut aussi être le lieu de certains traumatismes, incluant des abus, du rejet ou de l'intimidation. Dans le cas qui nous préoccupe ici, ces traumatismes viendraient plus de l'exposition aux comportements suicidaires des proches ou de la participation à d'éventuels pactes de suicide. Dans le premier cas, ce sont alors plus des mesures de postvention qui entreraient en jeu si le comportement suicidaire a été létal.

Une recension des écrits indique qu'aucun programme n'est spécifique à la prévention du suicide en milieu scolaire primaire, si ce n'est pour l'utilisation particulière des marionnettes avec des enfants (Sattem, 1990), et il n'existe donc pas non plus d'écrits sur l'évaluation d'une telle expérience. Le programme Intervention-suicide représente alors, en soi, une avancée dans le domaine puisque, notamment dans son Guide à l'intention des intervenants, il expose les principes de base qui peuvent guider la démarche. L'auteur du guide, soit le responsable du programme Intervention-suicide, s'y fait par contre très prudent puisqu'il est bien conscient que peu de recherches scientifiques appuient ses dires, notamment en ce qui concerne une éventuelle grille d'évaluation de l'urgence suicidaire chez les enfants.

L'objet de la présente recension s'est donc étendu à l'ensemble de la prévention du suicide en milieu scolaire, ce qui signifie, en pratique, que l'on s'intéresse surtout alors à la prévention au secondaire plutôt qu'au primaire (Steele et al., 2007 ; Gould et al., 2003; Burns et al., 2000). Il y a des différences très importantes entre les deux clientèles, mais des ressemblances existent aussi, ne serait-ce que dans l'organisation des services. Quoi qu'il en soit, il n'y a pas non plus beaucoup de recherches concernant le secondaire, du moins en ce qui se rapporte aux activités de postvention (Leenaars et al., 2005). Pour ce qui est plus particulièrement de la situation au Québec, un groupe de travail (Séguin et al., 2004) y a élaboré un programme déjà en appli- cation au secondaire: le Programme de postvention en milieu scolaire.

Quant à la promotion ou à la prévention en milieu scolaire, plus précisément pour l'augmentation des facteurs de protection chez les jeunes, Boyer (2001) rapporte aussi l'évaluation d'un programme de sensibilisation à la dépression testé au Québec: Solidaires pour la vie. Mais, effectivement, ce programme ciblait la dépression plutôt que le suicide.

De façon générale, Leenaars (2005) conclut à l'efficacité de la prévention du suicide en milieu scolaire. Par contre, l'Avis scientifique sur la prévention $d u$ suicide chez les jeunes (Julien et al., 2004) était plus circonspect à ce niveau. On sait que le débat là-dessus n'est pas clos (Steele et al., 2007), notamment au Québec où un Groupe de travail du CRISE s'est aussi penché sur la question. Pour information, rappelons que Julien et al. (2004, p. 28) concluaient, dans un contexte où, disaientils, "certaines études éveillent des soupçons quant aux effets négatifs possibles de quelques stratégies », avec douze recommandations dont six touchaient le milieu scolaire: 1) mettre en œuvre les mesures visant le développement des habiletés d'adaptation des jeunes; 2) faire preuve de prudence dans la mise en œuvre des activités de postvention; 3) ne mettre en œuvre aucune activité de sensibilisation à la réalité du suicide qui s'adresse à des groupes de jeunes, en raison des résultats préoccupants qui ont été observés chez des jeunes vulnérables; 4) faire preuve de prudence dans la mise en œuvre des programmes d'aide par les pairs ; 5) encourager le développement de réseaux de sentinelles dans les milieux de vie des jeunes; 6) soutenir la mise en œuvre de programmes à multiples composantes, destinés à promouvoir la santé mentale des jeunes, à condition qu'ils ne comportent pas d'activités de sensibilisation qui s'adressent à des groupes de jeunes.

Notons tout de suite que, dans le cas $\mathrm{du}$ programme Intervention-suicide en milieu scolaire primaire qui est évalué ici, une grande prudence semble avoir mené à son implantation (recommandation $n^{\circ} 2$ de Julien et al., 2004). Les pairs, surtout à cet âge, n'y sont pas sollicités (rec. $\left.n^{\circ} 4\right)$. Le programme prend bien soin de ne pas faire de sensibilisation auprès des élèves eux-mêmes (rec. $\mathrm{n}^{\circ} 3$ ) et il utilise effectivement le personnel scolaire (enseignants, secrétaires, concierges...) comme sentinelles auprès des jeunes (rec. $\mathrm{n}^{\mathrm{o}} 5$ ). Toutefois, le programme lui-même n'inclut pas d'activités de développement des habiletés (rec. $\mathrm{n}^{0} 1$ ) ou de promotion (rec. $n^{\circ} 6$ ). Signalons que le responsable du programme Intervention-suicide, par 
sa position à la DSP Gaspésie - Îlesde-la-Madeleine et dans les réseaux de la prévention du suicide au Québec, est éminemment informé de ces orientations et des débats qui les entourent ${ }^{2}$.

\section{ÉVALUATION DES FORMATIONS}

\section{OU SENSIBILISATIONS}

Le devis de l'évaluation d'implantation qui est présenté ici prévoyait l'évaluation des formations offertes aux intervenants du programme et des sensibilisations plus courtes offertes à l'ensemble du personnel scolaire. Or, les écrits qui existent dans le domaine ne concernent pas nécessairement la prévention du suicide ou le milieu scolaire. Plus précisément, toute une littérature s'intéresse de plus en plus à l'évaluation des programmes de sensibilisation offerts aux «sentinelles », soit « [...] des adultes susceptibles d'être en contact avec des personnes suicidaires par leur travail (par exemple les enseignants), leurs activités bénévoles (dans un club social, entre autres), la place qu'ils occupent dans leur milieu ou leur communauté (comme les animateurs), ou pour leurs qualités d'aidants naturels (Roy et al., 2006, p. 29). Selon Roggenbaum et al. (2007), il y aurait quatre types de programmes de sentinelles, du moins aux États-Unis: 1) le QPR (Question, Persuade, Refer), 2) le ASSIST (Living Works), 3) le Lifelines et 4) leur propre programme, soit le Florida Suicide Prevention Gatekeeper Training. Quant à eux, Matthieu et al. (2007) ont fait une analyse de contenu de six programmes reconnus sur le site américain du Suicide Prevention Resource Center (dont le QPR). Ils ont observé que tous les programmes ont des objectifs au regard 1) des connaissances, 2) des attitudes, 3) des croyances reliées à la demande d'aide et 4) des habiletés à intervenir, à demander de l'aide et à référer.

Rudd et al. (2006) notent avec justesse que, pour évaluer ce type de programme, il ne faut pas seulement vérifier si les participants se souviennent bien, par exemple, des signes de manifestations suicidaires. Il faut aussi vérifier si, lorsque l'occasion se présentera, ils seront confiants et disposés à agir en conséquence, soit à repérer les sujets ciblés et à les référer. Cerel et al. (2007) ont évalué 3958 sentinelles formées avec le modèle QPR (Quinnett, 2005). Les séances de formation des sentinelles duraient de 30 minutes à trois heures, selon les régions. En comparant l'état des participants avant et après la formation, ils ont pu documenter une amélioration significative au niveau de la perception qu'ils avaient de leurs connaissances, mais aussi de leur efficacité à mettre en œuvre ce qu'ils avaient appris. Selon Roggenbaum et al. (2007), il faut vérifier, du moins pour les séances de sensibilisation offertes aux sentinelles, s'il y a: 1) des changements d'attitudes (autorapportés) concernant l'intervention, 2) une augmentation des connaissances sur le suicide et 3) des changements réels de comportements (au niveau du repérage et de la référence). Dans l'évaluation qu'ils ont faite de leur programme, ils ont trouvé des améliorations à tous les niveaux (différences entre le prétest et le post-test). Trois mois plus tard cependant, les progrès s'étaient grandement atténués même s'ils étaient toujours supérieurs au niveau de base.

DiCara et al. (2006) ont évalué le programme Lifelines dans douze high schools du Maine aux États-Unis. Cependant, leurs résultats ne sont pas nécessairement toujours transposables ici, étant donné que le programme Lifelines inclut des pairs aidants (élèves du secondaire) lors des sensibilisations et que les gens sensibilisés sont même invités à réaliser des interventions ensuite (ce qui est contraire à la pratique du programme Interventionsuicide). Néanmoins, les questionnaires d'évaluation que DiCara et collaborateurs ont construits sont relativement bien faits et certains items ont pu être réutilisés ici pour l'évaluation du programme Intervention-suicide (DiCara, 2006, communication personnelle). Pour évaluer des séances de sensibilisation, une équipe de l'Université de Rochester a aussi développé un Survey of knowledge, attitudes, and gatekeeper behaviours for suicide prevention in schools (Wyman et al., 2006), dont certaines parties ont inspiré la méthode utilisée ici. L'évaluation de leur propre programme, semblable au QPR (Quinnett, 2005), a révélé des résultats très positifs. Au Québec, Chagnon et al (2007) ont évalué un programme de formation de trois jours offert à des aidants des milieux scolaires, communautaires ou institutionnels. Leur évaluation a démontré chez eux une augmentation significative au niveau des connaissances, des attitudes, et des habiletés d'intervention.

Pour ce qui est de l'évaluation des formations et des sensibilisations offertes dans le cadre du programme Intervention-suicide, nous nous sommes beaucoup appuyés sur l'article de Chagnon et al. (2007) et, plus précisément, sur la recension déjà présentée par Marcoux (2004). Cette chercheure elle-même s'était inspirée de divers travaux déjà réalisés par des collègues du CRISE, plus particulièrement Brian Mishara, François Chagnon et Sylvaine Raymond, mais aussi de Tierney (1988) et de Neimeyer et al. (1994). Il s'agit ici d'une approche dite "par compétences» plutôt que «par objectifs ». La compétence renvoie ici à des connaissances qu'il faut avoir ou acquérir (aspect cognitif), mais aussi à des aptitudes (dispositions naturelles ou acquises). Le deuxième aspect correspond plus à des caractéristiques individuelles. Plus spécifiquement, on distingue trois composantes à une compétence: le savoir (les connaissances), le savoir-faire (les habiletés) et le savoir-être (caractéristiques personnelles, valeurs, attitudes, croyances, estime de soi, traits de personnalité). Marcoux (2004) s'inspire aussi du modèle de Kirkpatrick (1967) qui précise qu'il y a quatre objets d'évaluation pour de telles formations: 1) les réactions à la formation (dont la satisfaction), 2) les apprentissages (connaissances, habiletés, attitudes), 3) le transfert des apprentissages en comportements d'intervention et 4) les impacts organisationnels. Marcoux (2004) suggère finalement quatre modes d'évaluation des compétences : 1) observation in vivo, 2) simulation, 3) tests objectifs et 4) auto-évaluation.

\section{MÉTHODE}

L'évaluation présentée ici est essentiellement une évaluation de l'implantation, mais, en complément, elle a aussi la prétention de donner quelques indices sur les éventuels effets du programme Intervention-suicide. Une telle évaluation se devait de demeurer "formative» plutôt que "sommative», en ce sens qu'elle ne devait pas trop s'intéresser à estimer la somme de ses effets et impacts sur les enfants ou sur la communauté. Effectivement, la démarche d'évaluation s'est donc intéressée longuement à développer différents modèles théoriques pour ce programme, modèles qui ont permis une meilleure compréhension du processus d'intervention actif dans ce programme, de même que de ses effets possibles. Notons aussi au passage que le programme sous étude est relativement jeune, du moins dans le sens que sa programmation était encore en remaniement lors du début des premières observations de l'évaluateur ${ }^{3}$ en avril 2006, et que ses activités n'étaient pas encore implantées dans plusieurs écoles.

Les résultats présentés ici proviennent essentiellement d'analyse de la documentation interne de l'organisme promoteur du programme, d'observation des activités (organisation, formation des intervenants, sensibilisation du personnel scolaire, interventions de groupe lors de postventions), d'entretiens avec des personnes concernées par le programme (directeurs de commissions scolaires, coordonnateurs des services éducatifs, directeurs d'école, intervenants du programme), et de questionnaires complétés par différents acteurs du programme (intervenants, enseignants 
et autres membres du personnel scolaire). Les questionnaires remplis par les intervenants qui participaient à des formations ont été inspirés des travaux de Marcoux (2004). Les questionnaires remplis par le personnel scolaire lors des séances de sensibilisation ont été inspirés des travaux de DiCara et al. (2006), Quinnett (2005), Rodgers (2007) et Wyman et al. (2006). Dans les deux cas, il s'agissait essentiellement de questionnaires où les répondants inscrivaient leur réponse sur une échelle Likert en quatre ou dix points, selon le contexte. Les questionnaires visaient à documenter la satisfaction des participants par rapport au contenu et au format des rencontres, mais surtout par rapport à l'amélioration éventuelle de leur motivation, de leurs connaissances spécifiques, de leurs habiletés et de leurs attitudes. Toute la collecte de données a été réalisée entre avril 2006 et juin 2007.

\section{RÉSULTATS ET DISCUSSION}

Les différents éléments de l'évaluation sont regroupés ici sous douze rubriques, à partir de ceux qui relèvent plus de la structuration du programme (documentation, implantation...) jusqu'à ceux qui sont plus de l'ordre des interventions réalisées. Par la suite, les différentes forces et faiblesses sont mises en relief. Il s'agit évidemment de l'état de la situation en juin 2007. Diverses recommandations, lesquelles ne sont pas rapportées ici, ont en effet permis de corriger la plupart des faiblesses.

\section{ANALYSE DE LA DOCUMENTATION \\ DU PROGRAMME}

Sur le plan formel, les trois guides réalisés pour ce programme sont exemplaires. Malgré la grande qualité de leurs contenus cliniques, il faut cependant constater qu'on n'y retrouvait pas vraiment de présentation formelle du programme, du moins en termes d'objectifs, de structure ou de modèles théoriques. Parallèlement à cela, les séances de sensibilisation du personnel scolaire ne sont pas suffisamment documentées, ni formellement construites en termes d'objectifs très spécifiques ou de changements à amener chez les participants. La documentation du programme n'aborde pas non plus le partage des véritables tâches cliniques d'intervention entre les différents types de professionnels des Commissions scolaires (CS) ou des Centres de santé et services sociaux (CSSS) impliqués dans ce programme: agents de ressources humaines et travailleurs sociaux professionnels $(25,93 \%$ de l'ensemble), psychologues (16,67\%), psychoéducateurs $(14,81 \%)$, éducateurs spécialisés (14,81\%), infirmiers $(14,81 \%)$, intervenants sociaux avec diplôme collégial (7,41\%), conseillers d'orientation $(3,70 \%)$ ou conseiller en rééducation $(1,85 \%)$.

\section{STRUCTURE ET IMPLANTATION}

DU PROGRAMME

L'implantation elle-même du programme s'est réalisée avec quelques retards, mais l'opération était terminée lors du dépôt du rapport d'évaluation. Ce programme décentralisé articule bien les trois niveaux d'activité les plus importants, soit celui des directeurs d'école, celui de l'ensemble du personnel scolaire et celui des intervenants membres des équipes de secteur. Les diverses personnes interviewées apprécient grandement la façon dont le programme a été structuré et implanté. Cependant, le responsable du programme n'a pas l'autorité nécessaire pour résoudre certaines difficultés de gestion, dont la mauvaise diffusion de l'information auprès des directeurs d'école. Ces difficultés sont liées à l'absence de partage formel des responsabilités, d'où la nécessité d'une plus grande formalisation de la concertation entre les trois niveaux d'instances qui sont parties prenantes d'un tel programme: la Direction de la santé publique, les Commissions scolaires (CS) et les Centres de santé et service sociaux (CSSS).

\section{ACTIVITÉS RÉALISÉES}

\section{PENDANT LA PÉRIODE D'ÉVALUATION}

Le nombre d'interventions réalisées dans chacun des cinq secteurs géographiques n'est pas bien comptabilisé, que ce soit en fonction du nombre ou de la diversité, ce qui ne rend probablement pas justice au travail qui est fait dans le cadre du programme. Quoi qu'il en soit, le nombre d'activités d'intervention pourrait probablement être augmenté par une intensification des activités de repérage dans les écoles à la suite des séances de sensibilisation ayant été réalisées pendant la durée de l'évaluation.

\section{CARACTÉRISTIQUES DES ÉLÈVES}

ET DES FAMILLES

Il n'y a pas vraiment de données fiables permettant de bien décrire le profil de la clientèle du programme. Pour ce qui est de la quantité d'enfants dont il est question ici, les seules statistiques disponibles sont celles produites lors des rencontres bilan des équipes et celles-ci ne sont pas nécessairement très justes. Il est également assez difficile d'établir quelle est la couverture réelle du programme, en termes de rapport entre le nombre d'enfants touchés par le programme et le nombre d'enfants en besoin.
CARACTÉRISTIQUES DES MEMBRES DES ÉQUIPES D'INTERVENTION

Les membres des équipes du programme, dans les différents secteurs géographiques, sont des professionnels de l'intervention psychosociale, si l'on excepte le cas particulier des infirmiers dont ce n'est pas le premier champ d'expertise. Il s'agit d'employés déjà en place dans les CSSS ou les $\mathrm{CS}$ et qui ne consacrent qu'une partie de leur temps au programme. Comme dans bien des domaines de l'intervention et de l'éducation, ce sont majoritairement des femmes. Les trois quarts des membres des équipes ont un diplôme universitaire et ils ont un bon bagage d'expérience pouvant être utilisé à l'intérieur du programme Intervention-suicide.

\section{CARACTÉRISTIQUES}

\section{DU PERSONNEL SCOLAIRE}

La grande majorité du personnel scolaire est composée d'enseignants féminins, le plus souvent avec diplôme universitaire. Leur moyenne d'âge est de 39 ans et ils ont en moyenne 14 ans d'expérience. À la suite de leur participation aux séances de sensibilisation organisées dans le cadre du programme, on s'attend à ce qu'ils soient capables de repérer les enfants en difficulté pour ensuite les référer aux intervenants du programme. En fait, ils s'estiment euxmêmes assez près des élèves et $66 \%$ d'entre eux ont, dans la dernière année, repéré au moins une fois un élève en détresse. Ils ne sont pas eux-mêmes considérés comme des «intervenants» dans le cadre du programme.

\section{RENCONTRES DE BILAN ET MISE À JOUR}

Dans chaque secteur géographique, des rencontres bisannuelles des intervenants permettent la distribution de la documentation la plus récente, des discussions et suivis sur les échanges entre les différentes instances du programme, la rétroaction et l'analyse des interventions réalisées. En 2006, les rencontres ont notamment permis de réactiver le programme. L'animation est relativement efficace et l'information clinique qui y est véhiculée est très appréciée des participants. En parallèle, ces rencontres permettent d'identifier certaines résistances du milieu qui peuvent justement être reliées aux difficultés de gestion du programme.

\section{SÉANCES DE FORMATION INITIALE DES INTERVENANTS}

La durée des séances de formation initiale a été réduite en 2006, ce qui a amené une certaine insatisfaction chez les nouveaux intervenants. La satisfaction générale des participants aux formations reste 
néanmoins très élevée, notamment par rapport au formateur lui-même. Quant à la motivation des participants, elle était déjà très élevée au point de départ, mais elle s'est également accrue après les séances de formation. Dans l'ensemble, les connaissances, habiletés et attitudes des participants se sont également améliorées d'environ $10 \%$.

\section{SÉANCES DE FORMATION SPÉCIALISÉE DES INTERVENANTS}

En supplément à la formation initiale, trois séances de formation portant sur l'intervention et la thérapie pour enfants endeuillés par suicide ont été dispensées en 2007 aux intervenants du programme. Les observations réalisées lors de l'une de ces séances révèlent une grande qualité tant au regard du contenu que du format des présentations. L'évaluation de la satisfaction face à cette formation est généralement très élevée, et ce, pour l'ensemble des éléments. En revanche, les éléments relatifs à l'auto-évaluation des habiletés acquises sont un peu moins élevés, révélant chez les intervenants certaines craintes dans la perception de leurs capacités. Cependant, presque tous se disent mieux outillés qu'avant. Dans l'ensemble, ce type de formations plus spécialisées, autant que d'autres qui pourraient devenir disponibles, est donc un atout pour le programme, ce qui justifie qu'elles soient désormais intégrées dans les formations de base.

\section{SÉANCES DE SENSIBILISATION \\ DU PERSONNEL SCOLAIRE}

L'organisation des séances de sensibilisation n'a pas été sans difficultés dans certains secteurs, le tout étant encore relié au problème de la répartition des responsabilités. D'un secteur à l'autre, on observe une disparité dans les contenus présentés et la façon de transmettre les informations n'est pas toujours heureuse malgré des initiatives locales très intéressantes. Néanmoins, les taux de satisfaction des participants à ces séances sont généralement assez élevés. Surtout au niveau des connaissances acquises, mais aussi des habiletés, des attitudes et du transfert des connaissances, les participants se sont grandement améliorés.

\section{INTERVENTIONS INDIVIDUELLES}

L'évaluateur ne pouvait observer directement des interventions individuelles réalisées avec des enfants. Toutefois, certaines ont été portées à sa connaissance. Il n'est pas toujours évident ici de voir si les interventions auraient tout de même été réalisées en l'absence du programme et si elles l'auraient été avec la même qualité. Sur le deuxième point, on peut penser que les intervenants, avant l'implantation du programme, auraient été beaucoup moins équipés pour intervenir au plan des connaissances, des habiletés et des attitudes. C'est du moins ce qu'a révélé l'évaluation des formations qu'ils ont suivies. Dans l'ensemble, il semble donc que les procédures du programme assurent un cadre relativement souple pour la prise en charge des enfants en besoin. En même temps, il faut bien voir que les cas rapportés sont relativement rares, ce qui peut poser problème pour la continuité dans l'application du programme. Dans l'ensemble, les intervenants estimaient néanmoins que le programme Intervention-suicide leur avait facilité la tâche lors de ces interventions.

\section{INTERVENTIONS DANS UNE ÉCOLE \\ APRĖS UN SUICIDE}

Cette partie de l'évaluation a documenté quatre interventions réalisées dans des écoles après des décès relativement traumatisants pour les communautés locales. L'application du protocole du programme pour ces situations s'est généralement faite sans trop de problèmes malgré les craintes exprimées par certains intervenants. Là où des difficultés sont survenues, des réunions d'équipe ont généralement permis d'identifier les correctifs nécessaires. Le fait qu'il soit difficile d'obtenir rapidement une confirmation officielle d'un suicide, un élément difficile à corriger, demeure tout de même un irritant dans bien des interventions. En règle générale, le milieu scolaire est très satisfait des interventions réalisées et il se sent rassuré par cet encadrement.

Par ailleurs, au moment où l'évaluation a été réalisée, différentes forces et faiblesses ont été identifiées. La situation a cependant évolué depuis, à la suite des recommandations faites par l'évaluateur.

\section{FORCES DU PROGRAMME}

1. Le programme articule bien trois niveaux d'activité, celui des directeurs d'école, celui de l'ensemble du personnel scolaire et celui des intervenants membres des équipes de secteur. Un niveau ne pourrait être fonctionnel sans l'autre.

2. Le programme utilise les forces d'intervention déjà présentes localement dans les secteurs de la santé et de l'éducation. Il n'y a donc pas d'embauche supplémentaire.

3. L'ensemble des partenaires est motivé, engagé et diligent malgré quelques difficultés relatives au partage des responsabilités.

4. Le personnel scolaire et les intervenants sont qualifiés professionnellement et ils se sentent aptes à recueillir les confidences des enfants.
5. L'expertise et la motivation du responsable de programme (son créateur) est un atout majeur ${ }^{4}$.

6. Les trois guides du programme sont remarquablement bien faits (format, clarté, précision, par étapes), surtout au plan clinique, et ils peuvent s'adapter à d'autres situations que les manifestations suicidaires ou les interventions dans une école après un suicide.

7. Le programme est unique, original et spécifique aux enfants du primaire, ce qui n'existait pas auparavant.

8. Les formations offertes aux intervenants sont bien structurées, appréciées, efficaces et adaptées aux besoins des intervenants.

9. Les séances de sensibilisation du personnel scolaire sont relativement bien structurées et très efficaces.

10. La diversité des expertises et expériences des intervenants est notable.

11. Le programme est ouvert à l'auto-évaluation et à la critique externe.

12. Le programme fait preuve de modération dans l'interprétation du phénomène suicidaire chez les enfants et dans les actions à prendre individuellement ou dans les écoles, tout en restant constant dans la diffusion du message de base.

\section{FAIBLESSES DU PROGRAMME}

1. Le partage des responsabilités n'est pas suffisamment formalisé.

2. Il y a un manque d'information explicite dans les guides sur les objectifs, la structure et les fondements théoriques du programme.

3. Les formations offertes aux intervenants sont trop courtes.

4. Il y a dilution de l'expertise chez les intervenants, compte tenu du peu de manifestations suicidaires à traiter.

5. Il y a un certain isolement chez des intervenants lorsque les équipes de secteur ne sont pas très actives.

6. Il y a absence fréquente de leadership dans les équipes de secteur, notamment pour le suivi des rencontres et des formations.

7. Il y a un décalage dans la formation clinique entre les intervenants de diverses origines.

$$
\star \star \star
$$

"Il faut tout un village pour éduquer un enfant », est-il proclamé sur la façade d'une école de la Gaspésie. Dans ce village qui est toujours le nôtre, où que nous vivions, l'école représente une instance importante, une instance que le reste du village interpelle de plus en plus. Pour le cas particulier de la prévention du suicide chez les enfants, nous avons tenté justement, 
à l'occasion de la présente évaluation, de voir si un programme appliqué dans les écoles remplissait les conditions pour être capable de bien jouer son rôle.

Là-dessus, les conclusions sont relativement positives avec l'implantation du programme Intervention-suicide en milieu scolaire primaire en Gaspésie et aux Îlesde-la-Madeleine. Il est maintenant du ressort des autres membres du «village» que nous habitons tous de s'assurer qu'ils font leur part dans la résolution du problème pour que des jeunes «arrêtent d'avoir mal en dedans ». En s'appuyant sur une conception écologique des choses, il est en effet clair qu'un programme de prévention du suicide chez les jeunes doit avoir des assises chez les individus (enfants et adultes), les familles, les milieux de vie (écoles, quartiers...), les organismes communautaires, les services de soin (dont ceux de pédopsychiatrie), les lieux de formation et de diffusion des connaissances, les lieux de culture ainsi que les organismes gouvernementaux et paragouvernementaux. Là-dessus, notons que, dans l'état actuel des choses, le programme Interventionsuicide en milieu scolaire primaire rallie tout de même des membres provenant d'au moins trois instances: direction de la santé publique, commissions scolaires et centres de santé et service sociaux. En soi, cela est déjà exemplaire, compte tenu du peu de réseautage et de collaboration qui est souvent constaté en prévention du suicide.

\section{Bibliographie}

BOYER, R. (2001). "Évaluation normative du processus et analyse de l'intervention de sensibilisation à la dépression chez les adolescents des $4^{\mathrm{e}}$ et $5^{\mathrm{e}}$ secondaire du plan d'action «Solidaires pour la vie» de la Fondation québécoise des maladies mentales (FQMM) ", dans Actes de la journée sur la prévention du suicide chez les jeunes. Le modèle de la Colombie-Britannique. Un regard sur les pratiques québécoises, Québec, Ministère de la Santé et des Services sociaux, p. 33-39.

BURNS, J.M. et G.C. PATTON (2000). "Preventive interventions for youth suicide: A risk factor-based approach », Australian and New Zealand Journal of Psychiatry, vol. 34, p. 388-407.

CEREL, J., J. PADGETT, V. ROBBINS et B. KAMINER (2007). « Kentucky gatekeeper training for suicide prevention", dans Proceedings of the 40th Conference of the American Association of Suicidology, Washington DC, American Association of Suicidology.

CHAGNON, F., J. HOULE, I. MARCOUX et J. RENAUD (2007). «Control-group study of an intervention training program for youth suicide prevention ", Suicide and Life-Threatening Behavior, vol. 37, $\mathrm{n}^{\circ}$ 2, p. 135-144.

DAIGLE, M. (2007). Évaluation de l'implantation du programme Interventionsuicide en milieu scolaire primaire. Rapport d'évaluation, Gaspé, Direction de santé publique Gaspésie-Îles-de-la-Madeleine, disponible en ligne, $<$ http://www.agencesssgim.ca/fichiers/agence/Sante_publique/ Rapport_final_de_l_evaluation_de_l_implantation_du_programme_Intervention-suicide_ en_milieu_scolaire_primaire.pdf $>$.

D'AMOURS, Y. (2001). «Les contributions possibles de l'école à la prévention du suicide », dans Actes de la journée sur la prévention du suicide chez les jeunes. Le modèle de la Colombie-Britannique. Un regard sur les pratiques québécoises, Québec, Ministère de la Santé et des Services sociaux, p. 25-32

DICARA, C., S. O'HALLORAN, M. MADDEN, D. HALEY, J. KALAFAT et K. LUBELL (2006). "Evaluation of a school based suicide response program ", dans 39th American Association of Suicidology Annual Conference, Washington DC, American Association of Suicidology.

GOULD, M.S., T. GREENBERG, D.M. VELTING et D. SHAFFER (2003). "Youth suicide risk and preventive interventions: A review of the past 10 years ", Journal of the American Academy of Child and Adolescent Psychiatry, vol. 42, p. 386-405

JULIEN, M. (2003). «La prévention du suicide chez les jeunes, un aperçu sommaire des études évaluatives », Revue québécoise de psychologie, vol. 24, $\mathrm{n}^{\circ}$ 1, p. 201-226.

JULIEN, M. et J. LAVERDURE (2004). Avis scientifique sur la prévention du suicide chez les jeunes, Québec, Institut national de santé publique du Québec.

KIRKPATRICK, D.L. (1967). « Evaluation of training », dans R.L. CRAIG et L.R. BITTEL (dir.), Training and Development Handbook, Berkeley (CA), McGraw-Hill, p. 87-112.

LEENAARS, A.A. (2005). «Effective public health strategies in suicide prevention are possible: A selective review of recent studies ", Clinical Neuropsychiatry, vol. 2, $\mathrm{n}^{\circ} 1$, p. 21-31.

LEENAARS, A.A., D. LESTER et S. WENCKSTERN (2005). "Coping with suicide in the schools: The art and the research ", dans R.I. YUFIT et D. LESTER (dir.), Assessment, Treatment, and Prevention of Suicidal Behavior, Hoboken (NJ), John Wiley and Sons, p. 347-377.

MARCOUX, I. (2004). "L'évaluation des formations en intervention sur le suicide: concepts, mesure et enjeux ", dans B.L. MISHARA et F. CHAGNON (dir.), Évaluation de programmes en prévention du suicide, Québec, Presses de l'Université du Québec.

MATTHIEU, M., P. ROGERS et W. CROSS (2007). "Content analysis of suicide prevention programs ", dans Proceedings of the 40th Conference of the American Association of Suicidology, Washington DC, American Association of Suicidology.

MINISTÈRE DE LA SANTÉ ET DES SERVICES SOCIAUX. (2001). Actes de la journée sur la prévention du suicide chez les jeunes. Le modèle de la Colombie-Britannique. Un regard sur les pratiques québécoises, Québec, Ministère de la Santé et des Services sociaux.
NEIMEYER, R.A. et A.M. PFEIFFER (1994). "Evaluation of suicide intervention effectiveness », Death Studies, vol. 18, n 2 , p. 131166.

QUINNETT, P. (2005). QPR Gatekeeper Training for Suicide Prevention CD-ROM, Spokane (WA), QPR Institute.

RODGERS, P. (2007). «Evaluating suicide prevention programs", dans Proceedings of the 40th Conference of the American Association of Suicidology, Washington DC, American Association of Suicidology.

ROGGENBAUM, S.D. ET P.G. HARRINGTON (2007). «Florida gatekeeper training evaluation", dans Proceedings of the 40th Conference of the American Association of Suicidology, Washington DC, American Association of Suicidology.

ROY, F., L. CARDINAL, L. DESCHÊNES, N. DUCHARME, S. GÉRIN-LAJOIE, F. GODIN, G. MARTEL, A. ROCHON et É. ST-HILAIRE (2006). L'implantation de réseaux de sentinelles en prévention du suicide: Cadre de référence, Québec, Ministère de la Santé et des Services sociaux.

RUDD, D., L. BERMAN et M.M. SILVERMAN (2006). "Warning signs for suicide: Theory, research, and clinical implications », dans 39th American Association of Suicidology Annual Conference, Washington DC, American Association of Suicidology.

SATTEM, L. (1990). «Suicide prevention in elementary schools ", Death Studies, vol. 14, $\mathrm{n}^{\circ} 4$, p. 329-346.

SÉGUIN, M., F. ROY, M. BOUCHARD, R. GALLAGHER, S. RAYMOND, C. GRAVEL et R. BOYER (2004). Programme de postvention en milieu scolaire: Stratégies d'intervention à la suite d'un suicide, Montréal, Association québécoise de prévention du suicide.

STEELE, M.M. et T. DOEY (2007). "Suicidal behaviour in children and adolescents. Part 2: Treatment and prevention », Canadian Journal of Psychiatry, vol. 52, $\mathrm{n}^{\circ}$ 6., suppl. 1, p. 35S-45S.

TIERNEY, R.J. (1988). Comprehensive Evaluation for Suicide Intervention Training, thèse de doctorat, Calgary, University of Calgary.

WYMAN, P.A., C.H. BROWN, J. INMAN, W. CROSS, K. SCHMEELK-CONE, J.B. PENA et J. GUO (2006). «Randomized controlled trial of gatekeeper training: Impact on school staff », dans 39th Conference of the American Association of Suicidology, Washington DC, American Association of Suicidology.

\section{Notes}

1. Proverbe africain affiché sur la façade de l'école Esdras-Minville, Grande-Vallée, Gaspésie.

2. Ce responsable est désormais affecté dans une autre région du Québec.

3. Le premier auteur de cet article.

4. Comme dans bien des programmes, cette force peut être difficile à maintenir à long terme lors des changements de personnel. 